\title{
FEATURES OF PHARMACOTHERAPY OF HEPATIC ENCEPHALOPATHY MANIFESTATIONS IN LIVER CIRRHOSIS
}

\author{
Viktor Meretskyi ${ }^{1}$, Natalya Lobanets ${ }^{2}$ \\ ${ }^{1}$ Department of Pharmacology and Clinical Pharmacology of I.Ya. Horbachevsky Ternopil State Medical University \\ ${ }^{2}$ Department of Clinical Pharmacy of I.Ya. Horbachevsky Ternopil State Medical University
}

Meretskyi V., Lobanets N. (2015), Features of pharmacotherapy of hepatic encephalopathy manifestations in liver cirrhosis. Health Problems of Civilization, 2 (9), p. 31-34.

Summary: The aim of this work is to study the effect of drug mebikar on the clinical manifestations of hepatic encephalopathy in patients with liver cirrhosis. The study involved 34 patients with cirrhosis of different etiologies. The average age of the examined patients was $(48.5 \pm 0.9)$ years, that prevailed patients of working age, indicating the medical and social significance of the problem of early diagnosis and adequate treatment of cirrhosis. One of the most frequent complications of cirrhosis is hepatic encephalopathy. In addition to conventional clinical and laboratory findings in patients with liver cirrhosis, severities of hepatic encephalopathy were determined according to West-Haven criteria before and after treatment. In patients with liver cirrhosis was established the presence of latent or clinically expressed hepatic encephalopathy. The treatment of the control group of patients consisted of the following drugs: essential phospholipids, mixture of sorbitol and major ions, arginine glutamate, furosemide, verospiron, lactulose, amoxicillin trihydrate and lansoprazole. In the complex treatment of the main group of patients medicine mebikar was administered additionally. Analysis of the clinical manifestations of hepatic encephalopathy showed a marked improvement in patients who received additional treatment with mebikar. Specifically, the incidences of mood changes as well as anxiety decreased in this group on average of 38\% compared with those patients without an additional treatment with mebikar. Also, sleep disturbances in the main group was observed to be lower by $7.2 \%$ compared to those in the control group. Inclusion in the treatment of patients with liver cirrhosis, the drug mebikar - a daytime tranquilizer with anxiolytic properties reduces neurotic disorders, improves emotional state which may indicate a regression in the manifestations of hepatic encephalopathy thereby improving the quality of life of patients and thus substantiating an expedient inclusion of mebikar an anxiolytic drug to the complex therapy of patients with liver cirrhosis.

Keywords: hepatic encephalopathy, liver cirrhosis, psychometric test

\section{Introduction}

The problem of liver cirrhosis (LC) is one of the most actual in modern gastroenterology and liver cirrhosis is a common chronic disease of the digestive system. In economically developed countries cirrhosis is among the six leading causes of death of patients aged 35-60 years, accounting for 25 to 400 cases per 100 thousand of population. 2 million people die from viral cirrhosis (mainly associated with hepatitis B and C) and virusassociated hepatocellular carcinoma in the world every year. The mortality rate from cirrhosis due to alcohol abuse in developed countries is close to that of viral LC (Zhdan et al. 2010, Babak et al. 2010, Golubovskaya, Shkurba 2011).

Cirrhosis is the final stage of chronic hepatitis with unfavorable course, the outcome of the outflow of bile and blood from the liver or genetic conditioned metabolic defects and characterized by a significant reduction in liver functioning cells, pronounced fibrous reaction, restructuring of liver parenchyma and vasculature with portal hypertension development and the formation of structurally abnormal regenerative nodes. One of the most frequent complications of cirrhosis is hepatic encephalopathy (HE) neuropsychiatric disorders inverse syndrome that occurs in hepatocellular insufficiency and/or seen with extensive portal-systemic shunts and after portocaval shunt surgery. The main clinical manifestations are changes in personality, intelligence and language disorders, the development of anxiety and depression, disorientation, impaired consciousness and

Address for correspondence: Viktor Meretskyi, I.Ya. Horbachevsky Ternopil State Medical University, m.Voli, 1, Ternopil, 46001, phone: +38 099 2376857, e-mail: meretskyivm@gmail.com

Tables: 1 Figures: 1 References: 12 Full-text PDF www.hpc.edu.pl Copyright (C) Pope John Paul II State School of Higher Education in Biała Podlaska, Sidorska 95/97, 21-500 Biała Podlaska Indexation: Index Copernicus, AGRO, ProQuest, Polish Medical Bibliography, Polish Ministry of Science and Higher Education. This is an open-access article distributed under the terms of the Creative Common Attribution Non-commercial license (http://creativecommons.org/licenses/by-nc/3.0), which permits use, distribution and reproduction in any medium, provided the original works is properly cited, the use is non-commercial and is otherwise in compliance with the license. 
sleep, and in severe cases coma and death of the patient (Gavrysh 2014, Polunina, Maev 2010). It should be noted that hepatic encephalopathy is the criterion of decompensation and is associated with poor prognosis with decreased quality of life. The survival of patients with HE stage 0-1 at timely hospitalization and treatment approaches $100 \%$, with 2 stages to $60-75 \%$, and the 3-4 stage with an average of 30\%. Even mild HE can significantly disrupt everyday functions require additional material expenditures. In 2003, direct medical costs in the United States of hospital patients with worsening HE, were estimated at 932 million dollars (Vilstrup et al. 2014).

The aim of the work is to study the influence of drug mebikar on the clinical manifestations of hepatic encephalopathy in patients with liver cirrhosis.

\section{Material and methods}

The study involved 34 patients with LC of different etiologies. Examined patients were distributed by gender as follows: 8 patients women (23.5\%), 26 patients male (76.5\%). Ratio of women and men is 1:3.3. The average age of the examined patients was $(48.5 \pm 0,9)$ years, that prevailed patients of working age, indicating the medical and social significance of the problem of early diagnosis and adequate treatment of cirrhosis. Duration of disease reached more than 20 years. Most patients constituted group of duration up to 5 years (63.2\%). In the diagnosis of liver cirrhosis classification of the International Working Group and the World Congress of Gastroenterology in Los Angeles in 1994, with refinements by International Statistical Classification of Diseases and Related Health Problems 10th Revision, was used.

The diagnosis of cirrhosis was verified by clinical data (complaints, medical history, physical data) and laboratory and instrumental examination according to the protocols of care by specialty "Gastroenterology" of the Ministry of Health of Ukraine.

The division of patients was performed according to the type of treatment. Base treatment of the control group of patients (1) consisted of essential phospholipids 2 capsules three times a day, a mixture of sorbitol and major ions $200 \mathrm{ml}$ intravenously, arginine glutamate $40 \% 5 \mathrm{ml}$ with $0.9 \%$ sodium chloride 200 ml intravenously, $4 \mathrm{ml}$ of furosemide intravenous bolus, verospiron 2 tablets per day, lactulose $15 \mathrm{ml}$ per day, amoxicillin trihydrate $1000 \mathrm{mg}$ per day, lansoprazole $40 \mathrm{mg}$ per day.

In the complex treatment of the main group of patients (2) drug mebikar under the trade name "Adaptol" manufactured by "Olaynfarm" (Latvia) $500 \mathrm{mg} 2$ times a day was administered additionally. In the available literature there are reports of "Adaptol" correction of psychopathological manifestations and impact on the system "lipid peroxidation, antioxidant protection," which is a factor of the syndrome of endogenous intoxication (Lapshina et al. 2008). The formation of endotoxemia in liver damage depends partly on adaptive processes activity of the nervous system neurohormonal structures (Harchenko, Huberhryts 2009). In view of these facts, complex therapy with day tranquilizer with anxiolytic properties - mebikar, that does not cause muscle relaxation, mental disorders and compares favorably with "typical" tranquilizers considered appropriate (Lapshina et al. 2008). Chemical inertness and lack of addictive syndrome such properties of the drug are taken into account. The dynamics of the clinical picture was assessed 2 weeks after the combined treatment. The effectiveness of treatment was evaluated by the following criteria: clinical manifestations of hepatic encephalopathy, indicators of performance during psychometric test.

In addition to conventional clinical and laboratory findings in patients with liver cirrhosis, severities of hepatic encephalopathy were determined according to West-Haven criteria (Babak et al. 2013, Randolph et al. 2009, Weissenborn 2013) before and after treatment. Part of the method is the number connection test (NCT) psychometric test used to detect latent hepatic encephalopathy and to quantify the impairment of mental function in hepatic encephalopathy stage 1 and 2. The test is aimed at determining the speed of cognitive activity. Methodology of test consisted in series connection of the proposed combination of numbers over time, the unit of measurement was considered in seconds. According to the parameters of specified method each numeric value of the test is equivalent to severity of hepatic encephalopathy.

Results are given as mean \pm S.E.M. Multiple comparisons were evaluated using ANOVA followed by the Student's t-test. Probabilities less than 0.05 were considered significantly different.

\section{Findings and discussion}

In most patients with cirrhosis was diagnosed asthenovegetative syndrome - $91 \%$. It is manifested by general weakness $(91 \%)$, decreased performance $(89 \%)$, increased fatigue $(63 \%)$, psycho-emotional lability (91\%), sleep disturbance (56\%), and headache (78\%). 
As a result of examination in $21(61.8 \%)$ patients with a favorable course of cirrhosis it was found subcompensation stage (Class B by Child-Pugh score) (Table 1).

Table 1. The distribution of patients with cirrhosis depending on the stage of the disease compensation

\begin{tabular}{|c|c|c|c|}
\hline Stage of disease compensation & $\begin{array}{c}\text { Males, } \\
\mathrm{n}=26\end{array}$ & $\begin{array}{c}\text { Females, } \\
\mathrm{n}=8\end{array}$ & $\begin{array}{c}\text { Total, } \\
\mathrm{n}=34\end{array}$ \\
\hline $\begin{array}{c}\text { Subcompensation } \\
\text { (class B) }\end{array}$ & $16(61.5 \%)$ & $5(62.5 \%)$ & $21(61.8 \%)$ \\
\hline $\begin{array}{c}\text { Decompensation } \\
\text { (class C) }\end{array}$ & $10(38.5 \%)$ & $3(37.5 \%)$ & $13(38.2 \%)$ \\
\hline
\end{tabular}

Data about the dynamics of the main clinical manifestations of hepatic encephalopathy are shown in Figure 1.

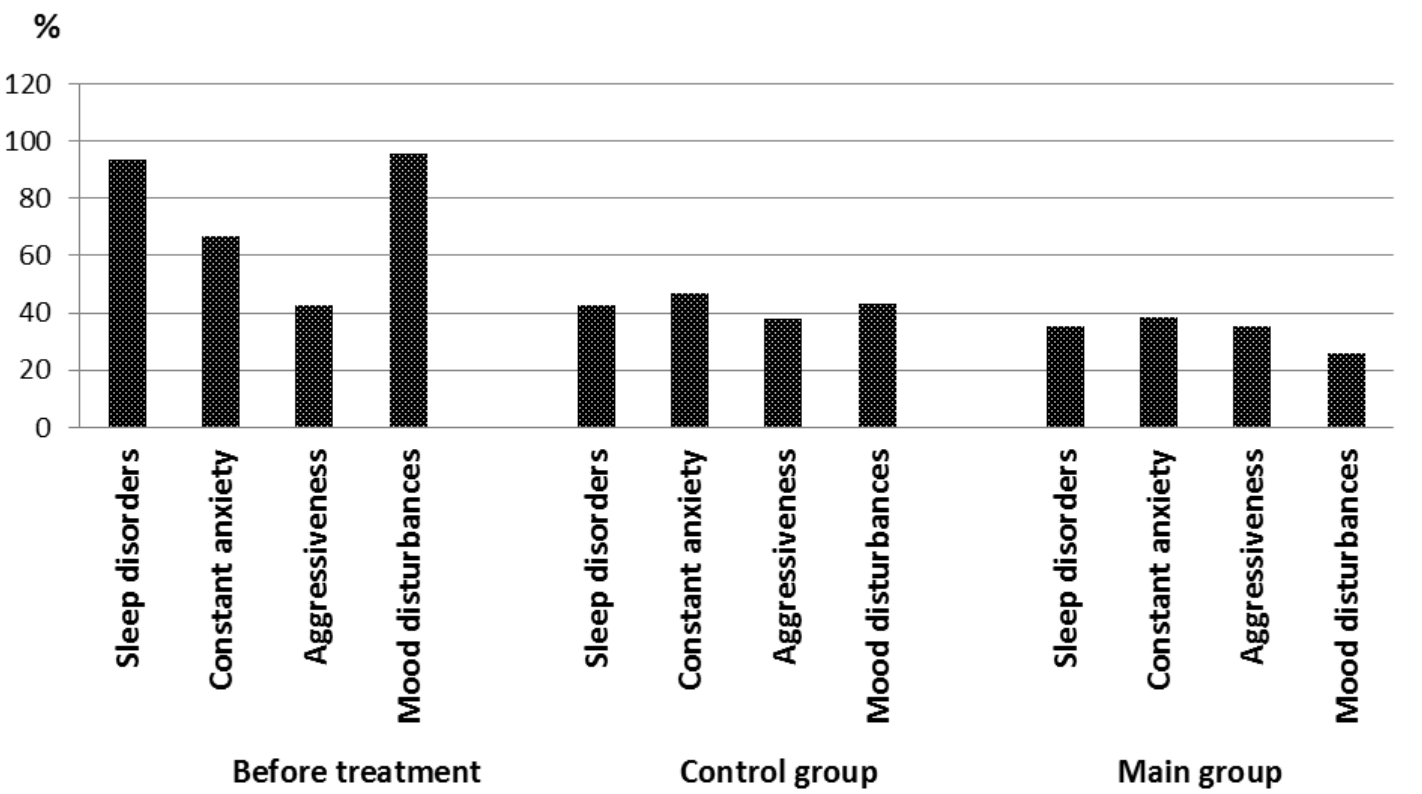

Figure 1. The dynamics expressions of hepatic encephalopathy indicators in patients with liver cirrhosis (\%)

It should be noted that in most patients, taking into account the results of the test, the 2 stage of hepatic encephalopathy was diagnosed. Most of the patients at the time of hospitalization had sleep and mood disturbances, less frequently noted ongoing unrest, in the smallest proportion of patients aggressiveness was observed. On average, patients performed the number connection test by $76.5 \pm 10,5$ seconds.

It was found the proportion of patients with the absence of clinical signs of hepatic encephalopathy 3 persons (8.8\%), the test showed changes characteristic for stage 1 encephalopathy in this group. Significant differences in the levels of hepatic encephalopathy and test in subgroups of women and men were not observed.

Analysis of clinical manifestations of hepatic encephalopathy symptoms showed a positive trend of evaluated indicators in both studied groups (Figure 1). However, a marked improvement was observed in patients who received additional mebikar under the trade name "Adaptol". Specifically, the incidences of mood changes as well as anxiety decreased in this group on average of $38 \%$ compared with those patients without an additional treatment with mebikar. Also, sleep disturbances in the main group was observed to be lower by $7.2 \%$ compared to those in the control group $(\mathrm{p}<0.05)$. Also found significant difference between the time of admission group and in the control group concerning mood disturbances and manifestation of aggressiveness in patients with liver cirrhosis (Figure 1).

The dynamics of treatment in both groups observed decrease of hepatic encephalopathy from stage 2 to stage 1 with a statistically significant difference before and after treatment in patients of the main group. By the severity of encephalopathy were appropriate psychometric test results. There was a tendency to decrease the duration of the test performance with more pronounced changes in the group of patients who received additionally mebikar. The choice was dictated by the drug mebikar features that distinguish it from benzodiazepine anxiolytics, such as lack of "behavioral toxicity", ie somnolence during the day, muscle relaxation, attention and motor coordination disorders, lack of dependence and limitations of physical and mental activity (Lapshina et al. 2008). 
So, hepatic encephalopathy is a frequent complication and one of the most debilitating manifestations of liver disease, severely affecting the lives of patients and their caregivers. Furthermore, cognitive impairment associated with cirrhosis results in utilization of more health care resources in adults than other manifestations of liver disease (Vilstrup et al. 2014). Hepatic encephalopathy produces a wide spectrum of nonspecific neurological and psychiatric manifestations. The mental (either cognitive or behavioral) and motor signs of HE may not be expressed, or do not progress in parallel, in each individual, therefore producing difficulties in staging the severity of HE. The neuropsychological test (NCT) is useful in the diagnosis of minimal HE and the assessment of cognitive function in patients with persistent HE (Cordoba 2014).

The study found that exposure to drug treatment with mebikar in patients with HE decreased incidence of aggression, constant anxiety and improved sleep and mood, increased rate of cognitive activity according to the number connection test.

\section{Conclusions}

In patients with liver cirrhosis was established the presence of latent or clinically expressed hepatic encephalopathy. Inclusion in the treatment of patients with liver cirrhosis, the drug mebikar - a daytime tranquilizer with anxioly tic properties reduces neurotic disorders, improves emotional state which may indicate a regression in the manifestations of hepatic encephalopathy thereby improving the quality of life of patients and thus substantiating an expedient inclusion of mebikar an anxioly tic drug to the complex therapy of patients with liver cirrhosis.

\section{References:}

1. Babak O., Kolesnikova E., Kozyreva T. (2010), Modern possibilities of correction of hepatic encephalopathy in patients with liver cirrhosis. Modern Gastroenterol. 4 (54), 71-76.

2. Babak O., Sytnik K., Kurinnaya E. (2013), Diagnosis and treatment of minimal hepatic encephalopathy. Ukrainian Journal of Therapeutics, 3, 81-86.

3. Cordoba J. (2014), Hepatic encephalopathy: from the pathogenesis to the new treatments. Hepatology. http:// dx.doi.org/10.1155/2014/236268.

4. Gavrysh I. (2014), Correction of hepatic encephalopathy in patients with cirrhosis combined with intestine dysbiosis. Bukovynskiy Medical Journal, 18, 1(69), 18-22.

5. Golubovskaya 0., Shkurba A. (2011), Modern approaches to correction of hepatic encephalopathy in patients with cirrhosis. Modern gastroenterol. 4(60), 120-123.

6. Harchenko N., Huberhryts N. (2009), Alcohol disease of the digestive system: clinical essays. Kiev: Novyi Druk.

7. Lapshina L., Kravchun P., Shevchenko O. (2008), Correction by adaptol of psychopathological manifestations and oxidative stress in patients with myocardial infarction. Medical Practice Activities, 1(61), 23-30.

8. Polunina T., Maev I. (2010), Hepatic encephalopathy. Algorithm of differential diagnosis and management tactics. Russian Medical Journal, 5, http://www.rmj.ru/articles_7018.htm.

9. Randolph C., Hilsabeck R., Kato A. (2009), Neuropscyhological assessment of hepatic encephalopathy: ISHEN practice guidelines. Liver Int. 29, 629-635.

10. Vilstrup H., Amodio P., Bajaj J. et al. (2014), Hepatic encephalopathy in chronic liver disease: 2014 Practice guideline by the American association for the study of liver diseases and the European association for the study of the liver. Hepatology, 60, 2, 715-735.

11. Weissenborn K. (2013), Psychometric tests for diagnosing minimal hepatic encephalopathy. Metabolic Brain Disease, 28 (2), 227-229.

12. Zhdan V., Babanina M., Kitura O. (2010), Gastroenterology in family physician practice: Tutorial. Poltava. 